\title{
End-to-End Learning of Visual Representations from Uncurated Instructional Videos
}

\author{
Antoine Miech ${ }^{1 *}$ \\ Ivan Laptev ${ }^{1}$ \\ Jean-Baptiste Alayrac ${ }^{2 *}$ \\ Josef Sivic ${ }^{1,3}$ \\ Lucas Smaira $^{2}$ \\ ${ }^{1}$ ENS/Inria ${ }^{2}$ DeepMind $\quad{ }^{3}$ CIIRC CTU $\quad{ }^{4}$ VGG Oxford \\ antoine.miech@inria.fr, jalayracegoogle.com \\ https://www.di.ens.fr/willow/research/mil-nce/
}

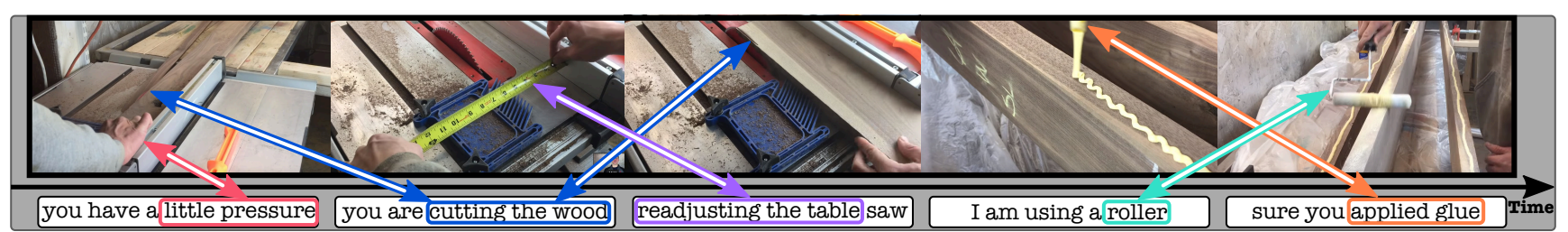

Figure 1: We describe an efficient approach to learn visual representations from misaligned and noisy narrations (bottom) automatically extracted from instructional videos (top). Our video representations are learnt from scratch without relying on any manually annotated visual dataset yet outperform all self-supervised and many fully-supervised methods on several video recognition benchmarks.

\begin{abstract}
Annotating videos is cumbersome, expensive and not scalable. Yet, many strong video models still rely on manually annotated data. With the recent introduction of the HowTol00M dataset, narrated videos now offer the possibility of learning video representations without manual supervision. In this work we propose a new learning approach, MIL-NCE, capable of addressing misalignments inherent in narrated videos. With this approach we are able to learn strong video representations from scratch, without the need for any manual annotation. We evaluate our representations on a wide range of four downstream tasks over eight datasets: action recognition (HMDB-51, UCF-101, Kinetics-700), text-tovideo retrieval (YouCook2, MSR-VTT), action localization (YouTube-8M Segments, CrossTask) and action segmentation (COIN). Our method outperforms all published selfsupervised approaches for these tasks as well as several fully supervised baselines. Our joint text-video pretrained model is publicly available at: https: //www. di.ens . fr/willow/research/mil-nce/.
\end{abstract}

\section{Introduction}

What we see changes what we know. What we know changes what we see. Jean Piaget
Vision and language play an important role in the way humans learn to associate visual entities to abstract concepts and vice versa. This has also become the de facto way to successfully train computer vision models. Indeed, from classification where images are categorized based on a fixed list of words to the recent captioning tasks where images or videos are annotated with rich language descriptions, this interplay is one of the driving forces behind recent progress in the field. However, one of the main limitations of this approach is that it requires manually annotating large collections of visual data.

Manual annotation is both cumbersome and expensive. Moreover, for videos, which are the main focus of this work, annotation is also even more challenging due to the ambiguities of choosing the right vocabulary of actions and annotating action intervals in video. This significantly limits the scale at which fully supervised video datasets can be obtained and hence slows down the quest to improve visual representations. Recent work has proposed a promising alternative to this fully supervised approach: leveraging narrated videos that are readily available at scale on the web.

Of particular interest, the recent HowTo100M dataset [52] contains more than 100 million pairs of

\footnotetext{
*Equal contribution.

${ }^{1}$ Département d'informatique de l'ENS, École normale supérieure, CNRS, PSL Research University, 75005 Paris, France.

${ }^{3}$ Czech Institute of Informatics, Robotics and Cybernetics at the Czech Technical University in Prague.

${ }^{4}$ VGG, Dept. of Engineering Science, University of Oxford
} 
video clips and associated narrations. It was automatically collected by querying YouTube for instructional videos. Such videos usually depict someone explaining orally how to perform a complex human activity, e.g. preparing a particular meal or repairing a car. Our objective in this paper is to learn strong video representations using only this narrated material.

End-to-end learning from instructional videos is a highly challenging task. Indeed, these videos are made in general with the goal of maximizing the number of views, and with no specific intention to provide a training signal for machine learning algorithms. This means that the supervision present in the narration is only weak and noisy. Among typical sources of noise, the principal one by far is the weak alignment between the video and the language: although for the most part the spoken words correlate with what is happening in these videos, this alignment is far from perfect. People might talk about something before actually demonstrating it, but they might also omit to talk about something that is happening because it is clear enough visually. Conversely they could only mention an action without showing it in the case where the step is not essential or trivial to convey with language alone. This is without even considering the irrelevant information given throughout the video (e.g. jokes or credits) as well as the general difficulty of working with spoken language obtained from potentially erroneous speech recognition algorithm as opposed to written text.

In this work, we propose a bespoke training loss, dubbed $M I L-N C E$ as it inherits from Multiple Instance Learning (MIL) and Noise Contrastive Estimation (NCE). Our method is capable of addressing visually misaligned narrations from uncurated instructional videos as illustrated in Figure 1. Equipped with this novel training scheme and a simple joint video and text embedding model, we show that we can successfully train video representations from scratch directly from pixels on the HowTo100M [52] dataset. To demonstrate the quality of the learnt representations, we employ an extensive set of evaluation benchmarks on a wide variety of video understanding tasks: action recognition (HMDB-51, UCF-101, Kinetics-700), text-tovideo retrieval (YouCook2, MSR-VTT), action localization (YouTube-8M Segments, CrossTask) and action segmentation (COIN). Notably, our learnt video representations outperform fully supervised baselines trained on Kinetics or ImageNet for several of the tasks. We also show improvements over other self-supervised approaches on HMDB51 and UCF101 even without fine-tuning the learnt representations. Finally, by leveraging the joint video and text representations, our off-the-shelf trained model also reaches state-of-the-art results on YouCook2 and CrossTask, without any training on the target datasets.

Contributions. The contributions of this work are threefold. (i) We propose a method to learn a joint text video embedding in an end-to-end fashion from unlabelled, uncurated narrated videos using the recently introduced HowTo100M [52] dataset. In particular, we introduce a specific loss, dubbed MIL-NCE for Multiple Instance Learning Noise Contrastive Estimation, that enables the learning to cope with the highly misaligned narration descriptions. (ii) We provide a thorough ablation study to quantitatively assess the importance of the different design choices of the approach. (iii) Finally, we demonstrate that the representations thus obtained are competitive with their strongly supervised counterparts on four downstream tasks over eight video datasets.

\section{Related work}

Learning visual representations from unlabeled videos. As labeling videos is cumbersome, expensive and not scalable, a significant number of prior works have studied the task of learning visual representations from unlabeled videos. Currently, the most effective approach is to collect a large amount of data from social media and use the available metadata as supervision $[1,26]$. However, this metadata is often in the form of keywords or tags, rather than (spoken) natural language considered in this work. In addition, the meta data is often platform dependent and rarely publicly available. Self-supervised approaches do not suffer from these issues as the idea is to define a supervised proxy task using labels directly generated from videos. Some of these tasks include: temporal ordering of video clips or frames [24, 46, 54, 86], predicting geometric transformations [37], maximizing the mutual information of multiple views [74], predicting motion and appearance [78], predicting the future, the past or a portion of masked input in the feature space [30, 71, 76], colorizing videos [77], predicting 3D geometry from synthetic data [25], predicting the audio in a feature space $[7,43]$ or tasks leveraging temporal cycle consistency $[23,81]$. In this work our proxy task is supervised by the automatic speech recognition (ASR) applied to narrated instructional videos. The nature of this supervision has the potential to also provide semantic information [52, 67], which is often missing in works that only exploit pixel-wise cues. Moreover, most of the top performing prior works only study their method on curated video datasets (e.g. Kinetics [14]) where labels have been removed. However, this is not truly learning from unlabeled data as these videos have been carefully selected and verified to belong to classes of interests. Caron et al. [12] further explain the performance gap between training on such curated data versus uncurated ones, truly available at scale. Instead, our approach focuses on the learning of representations only from uncurated videos.

Vision, speech and language. A common alternative to training visual models using manually defined sets of labels is to exploit semantic supervision from natural language or 


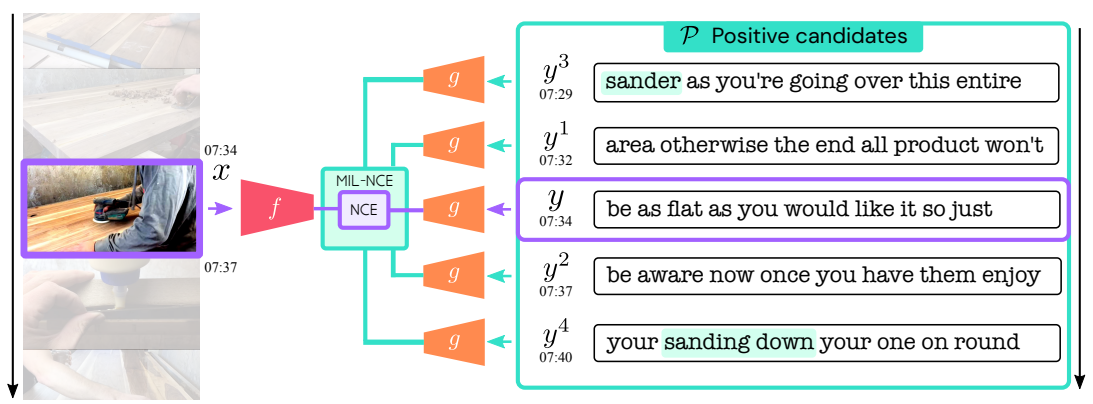

(a) Examples of positive candidates

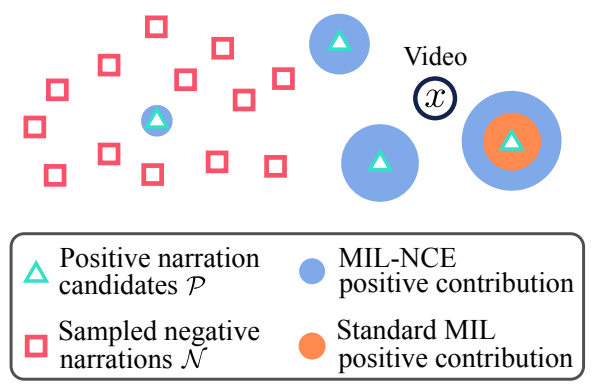

(b) Illustration of $M I L-N C E$

Figure 2: Left. Our MIL-NCE makes it possible to consider a set of multiple positive candidate pairs $\left\{(x, y),\left(x, y^{1}\right), \ldots,\left(x, y^{4}\right)\right\}$ while the standard NCE approach would only consider the single $(x, y)$ training pair and miss the visually grounded object description sander from pair $\left(x, y^{3}\right)$ or the action description sanding down from $\left(x, y^{4}\right)$. Right. Given a video $x$ and an associated set of positive narration candidates $\mathcal{P}$ (green triangles) that may or may not be correct, our MIL-NCE selects multiple correct positives (large blue areas) while downweighting incorrect positives (smaller blue areas) based on a discriminative ratio against negatives $\mathcal{N}$ (red squares). In contrast, traditional MIL considers only one positive (orange circle) while discarding the rest.

speech. Numerous prior works $[18,21,27,28,42,51,55$, $60,62,88,79,80,83,84]$ have used image / video description datasets $[48,63,65,87,90]$ to learn an embedding space where visual and textual data are close only if they are semantically similar. These methods either rely on manually annotated image / video description datasets, or leverage representations already pre-trained on manually labelled datasets (e.g. ImageNet [66] or Kinetics [14]). In contrast, in this work no manually annotated visual data is involved at any stage of our approach. To avoid labeling visual data, several approaches have leveraged audio transcripts obtained from narrated videos using automatic speech recognition (ASR) as a way to supervise video models for object detection [3, 15, 56], captioning [34, 72], classification [2, 44, 49, 89], summarization [59] or retrieval [52] using large-scale narrated video datasets such as How2 [67] or HowTo100M [52]. Others [10, 31] have investigated learning from narrated videos by directly using the raw speech waveform instead of generating transcriptions. Most related to us is the work of Miech et al. [52] who trained a joint video and text embedding from uncurated instructional videos [52]. However, as opposed to our work, they do not model any misalignment issue encountered when training on such videos and rely on visual representations pretrained on Kinetics-400 and ImageNet. Building on this work, Sun et al. [71] have used a contrastive bidirectional transformer (CBT) to learn long term contextual video representations from instructional videos. All these works use a visual representation pre-trained on either Kinetics or ImageNet when training on such narrated videos. In contrast, the key innovation of our work is that we demonstrate learning a generic video representation as well as a joint video-text embedding from scratch, without pre-training on manually annotated video or image datasets.
Multiple instance learning for video understanding. Multiple instance learning methods have been employed in many weakly-supervised video understanding problems including: person recognition in movies using scripts $[11,50$, 61], anomaly detection [70], weakly supervised action classification [47, 68] and localization [16, 22, 82], co-reference resolution of characters in TV series [64] or object tracking [8]. These methods often rely on some form of maxpooling (i.e. MIL-SVM [4]) or discriminative clustering (i.e. DIFFRAC [9]) to resolve the label ambiguities, and have used mostly linear (or shallow) models. In this work, we present MIL-NCE, a new approach marrying the noise contrastive estimation (NCE) framework [29] with multiple instance learning [20]. We show that MIL-NCE is wellsuited to learn deep visual representations from scratch using the weak and noisy training signals available in uncurated instructional videos.

\section{Leveraging Uncurated Instructional Videos}

This section describes the proposed approach to train joint video and text embeddings from unlabeled narrated videos in an end-to-end fashion. To start with, we are given $n$ pairs of video clips and associated narrations. In practice, a pair is composed of a short 3.2 seconds video clip (32 frames at 10 FPS) together with a small number of words (not exceeding 16) that correspond to what the person is saying in the video. For example, someone might be sanding wood while mentioning the action "sanding down" or the object "sander" as illustrated in Figure 2a. Given this input, our goal is to learn a joint embedding space where similarity between the narration and video embedding is high when the text and visual content are semantically similar and low otherwise, and we wish to learn this starting from raw pixels in the video and text descriptions. 
As illustrated in Figure 1, this is a very challenging problem due to the often severely misaligned visual descriptions.

In this work, we address this issue by introducing the MIL-NCE objective:

$$
\max _{f, g} \sum_{i=1}^{n} \log \left(\frac{\sum_{(x, y) \in \mathcal{P}_{i}} e^{f(x)^{\top} g(y)}}{\sum_{(x, y) \in \mathcal{P}_{i}} e^{f(x)^{\top} g(y)}+\sum_{\left(x^{\prime}, y^{\prime}\right) \sim \mathcal{N}_{i}} e^{f\left(x^{\prime}\right)^{\top} g\left(y^{\prime}\right)}}\right)
$$

where $x$ represents a video clip and $y$ a narration. $f$ and $g$ are the two embedding functions that respectively operate over video and text. Given a specific sample (indexed by $i$ ), we construct $\mathcal{P}_{i}$ to be a valid set of positive video/narration candidate pairs (see Figure 2) while $\mathcal{N}_{i}$ conversely refers to an associated set of negative video/narration pairs. This objective function implies maximizing the ratio of the sum of the positive candidate scores from $\mathcal{P}_{i}$ to the sum of the scores of all negatives sampled from $\mathcal{N}_{i}$, where the score is measured by the exponentiated dot product of the corresponding video and language embeddings, $f(x)$ and $g(y)$.

In the following, we describe more precisely the motivation behind the MIL-NCE objective (1). First, Section 3.1 introduces the chosen probabilistic model for joint text and video embedding. Given that model, Section 3.2 details the choice behind the training objective (1) explaining how it is specifically adapted to handle the misalignment noise inherent in narrated videos in comparison with existing approaches.

\subsection{A simple joint probabilistic model}

In the following, $x \in \mathcal{X}$ stands for a video clip and $y \in \mathcal{Y}$ for a narration. Given a set of $n$ pairs of video clips and associated narrations $\left\{\left(x_{i}, y_{i}\right)\right\}_{i=1}^{n} \in(\mathcal{X} \times \mathcal{Y})^{n}$ sampled from the joint data distribution $P(\mathcal{X} \times \mathcal{Y})$, our goal is to learn a joint embedding space where semantically related videos and texts are close and far away otherwise.

Formally, we learn two parametrized mappings: $f$ : $\mathcal{X} \rightarrow \mathbb{R}^{d}$ maps a video clip $x$ into a $d$-dimensional vector $f(x) \in \mathbb{R}^{d}$, and $g: \mathcal{Y} \rightarrow \mathbb{R}^{d}$ maps a narration $y$ into the same $d$-dimensional vector space, $g(y) \in \mathbb{R}^{d}$. We assume that we can estimate up to a constant factor the joint probability of a pair of video and narration $(x, y)$ by exponentiating the dot product of the two embeddings:

$$
p(x, y ; f, g) \propto e^{f(x)^{\top} g(y)} .
$$

In this work, $f$ takes the form of a CNN that runs over a fixed-length clip. For $g$, we consider simple sentence based models that transform a set of words into a single vector. Note, for simplicity and with a slight abuse of notation, we refer to $f$ (or $g$ ) as both a function and the parameters that define it. Also, we will refer to (2) as simply $p(x, y)$, i.e. we keep the dependence in $f$ and $g$ implicit for the clarity of simpler equations. More details about the exact architecture of the models are provided in Section 4.

\subsection{Learning from uncurated data: MIL-NCE}

Recall that our goal is to learn a joint video and text representation only from uncurated narrated videos. In this section, we start by detailing why this is a highly challenging endeavor due to misalignments present in that data. Next, we explain how the introduced MIL-NCE objective (1) enables to learn despite that noise. Finally, we contrast our proposed approach to similar works in the selfsupervised domain.

Misalignment in narrated videos. In [52], the authors estimate that around 50\% of clip-narration pairs from the HowTo100M dataset are not aligned. In fact, people are likely to describe an event after or before performing it in the video as illustrated in Figure 1. This temporal misalignment makes it more challenging to learn video representations than with manually annotated and aligned labels.

How to learn despite noisy supervision? To address the aforementioned issues, we propose to consider multiple options for matching a video and a narration instead of only comparing a single video $x$ with a single narration $y$ as done in [52]. Let's consider the example illustrated in Figure 2a. Given a clip $x, K$ narrations $\left\{y_{k}\right\}_{k=1}^{K}$ that happen close in time within the same video can be considered as positive candidates. By doing so, the chance that spoken words correlate with what is happening in the video increases. In that case, we would like to match at least one of the narrations $\left\{y_{k}\right\}_{k=1}^{K}$ with video $x$. Given the probabilistic model (2), a natural way to express this is by computing the joint probability of $x$ happening with any of the $y_{k}$. Because we can make the assumption that $y_{k}$ 's are mutually exclusive (i.e. $\left.\left(x, y_{i}\right) \neq\left(x, y_{j}\right), \forall i \neq j\right)$, this can be expressed mathematically by (2) as follows:

$$
p\left(\cup_{k}\left\{\left(x, y_{k}\right)\right\}\right)=\sum_{k} p\left(x, y_{k}\right) \propto \sum_{k} e^{f(x)^{\top} g\left(y_{k}\right)} .
$$

This is a MIL extension, which, as opposed to MIL-SVM, does not explicitly select a single positive sample per bag at training. More generally, and symmetrically, the case where several video clips are candidates for a given narration can also be envisioned. Hence, for generality, we assume that instead of having a single pair $(x, y)$, we have a set of candidate positive pairs $\mathcal{P}=\left(x^{k}, y^{k}\right)_{k=1}^{K}$, and we can simply repurpose (3) as $p(\mathcal{P}) \propto \sum_{(x, y) \in \mathcal{P}} e^{f(x)^{\top} g(y)}$. We denote by $\left\{\mathcal{P}_{i}\right\}_{i=1}^{n}$ the training set of candidate positive pairs deduced from the original training set $\left\{\left(x_{i}, y_{i}\right)\right\}_{i=1}^{n}$. With this extension, we have the tools to address misalignments. Details about how to construct $\mathcal{P}_{i}$ are given in Section 4.1

How to train this model? We wish to learn a video representation based on the previously described probabilis- 
tic model $p(\mathcal{P})$. However, this is challenging as one cannot directly apply standard generative techniques such as maximum likelihood due to the intractability of computing the normalization constant over all possible pairs of videos and narrations. Instead, we rely on a discriminative technique, namely the noise-contrastive estimation $(N C E)$ approach $[29,38]$, that has recently been shown to be effective in the context of feature learning $[32,57]$. The core idea is to directly optimize the unnormalized probabilistic model (3) to discriminate between data obtained from the true joint distribution $P(\mathcal{X} \times \mathcal{Y})$ and some artificially generated noise data, also called "negatives". In this work, we use the softmax version of NCE [38]:

$$
\max _{f, g} \sum_{i=1}^{n} \log \left(\frac{e^{f\left(x_{i}\right)^{\top} g\left(y_{i}\right)}}{e^{f\left(x_{i}\right)^{\top} g\left(y_{i}\right)}+\sum_{\left(x^{\prime}, y^{\prime}\right) \sim \mathcal{N}_{i}} e^{f\left(x^{\prime}\right)^{\top} g\left(y^{\prime}\right)}}\right)
$$

and replacing the probability of a single positive match, $e^{f\left(x_{i}\right)^{\top} g\left(y_{i}\right)}$, with our MIL like extension, $\sum_{(x, y) \in \mathcal{P}_{i}} e^{f(x)^{\top} g(y)}$, gives our proposed MIL-NCE training objective (1). Given this, we can simply estimate the parameters of our model by maximizing the objective (1), where $\mathcal{N}_{i}$ is a specific set of negatives for the $i$-th sample. Next, we discuss how our approach differs from prior work. NCE objectives for self-supervised learning. NCE has recently been successfully applied to self-supervised learning. In particular, CPC [32, 57] introduces the InfoNCE loss to enforce the model to maximize the conditional probability of some targets (e.g. the bottom part of the image) conditioned on some context (e.g. the top part of the image). Differently from CPC, which creates an asymmetric set of negatives by fixing the context and only sampling negative targets, we instead use NCE to model the symmetric joint probability between text and video (2). Thus, we construct $\mathcal{N}_{i}$ so that it contains both negatives for video $x_{i}$ and narration $y_{i}$. In Section 4 , we describe how $\mathcal{N}_{i}$ is obtained as well as evaluate the benefit of this symmetric approach.

\section{Experiments}

We first describe implementation details of our method in Section 4.1. The datasets used in our evaluation are outlined in Section 4.2. We present an ablation study emphasizing key ingredients of our approach in Section 4.3. Finally, we compare our learnt representations to previous self and fully-supervised methods in Section 4.4.

\subsection{Implementation details}

Model and Inputs. For the 3D CNN backbone, we use the standard I3D implementation from [14] for all ablation studies and for the comparison to state-of-the-art, we report result on both I3D and S3D [85]. We use the Google News self-supervised pre-trained word2vec $(\mathrm{d}=300)$ embedding

\begin{tabular}{|c|c|c|c|}
\hline \multirow{2}{*}{\multicolumn{2}{|c|}{ 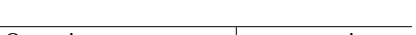 }} & \\
\hline & & Operation & output size \\
\hline Input video & $32 \times 200 \times 200 \times 3$ & Embedding & $16 \times 300$ \\
\hline I3D / S3D $\rightarrow$ Mixed_5c & $4 \times 6 \times 6 \times 1024$ & Linear + ReLU & $16 \times 2048$ \\
\hline Global avg pool & $1 \times 1 \times 1 \times 1024$ & Max pool & $1 \times 2048$ \\
\hline Linear & $1 \times 1 \times 1 \times 512$ & Linear & $1 \times 512$ \\
\hline
\end{tabular}

Table 1: Video (left) and text (right) model architectures.

from [53] for our word representation. Each video clip at training contains 32 frames sampled at 10 fps (3.2 seconds) with a 200x200 resolution (224x224 at test time). For each narration, we take a maximum of 16 words. More details about the model architecture and input dimensions are provided in Table 1. A detailed illustration of the architecture is also given in Appendix (section B).

Visual representations evaluation. We evaluate our visual representations at two different semantic levels. First, we use the output of the I3D (or S3D) Global avg pool (see Table 1), to evaluate our representation for action recognition, action segmentation and action localization. Next, the output of the last I3D (or S3D) Linear layer (see Table 1), which maps the video to the joint text-video semantic space, is used in conjunction with the output of the language model for the text-video retrieval tasks.

Training dataset. We train our model using the HowTo100M [52] narrated video dataset. It consists of more than $1.2 \mathrm{M}$ videos accompanied with automatically generated speech transcription. We use the provided transcription to create pairs of video / caption defined by each caption time stamp. Note that while the original dataset [52] consists of $136 \mathrm{M}$ pairs, we only used $120 \mathrm{M}$ pairs to comply with the YouTube wipe out policy. Each video shorter than 5 seconds is extended symmetrically in time so that the duration is at least 5 seconds. Then we randomly sample, a fixed length clip of 3.2 seconds within each video at training. For each clip-narration training pair $(x, y)$ sampled, we construct the bag of positive candidate pairs $\mathcal{P}$ by considering the nearest captions in time to $y$ as depicted in Figure 2a. For example, if we set the number of positive candidate pairs to 3 , we would have $\mathcal{P}=\left\{(x, y),\left(x, y^{1}\right),\left(x, y^{2}\right)\right\}$ where $y^{1}$ and $y^{2}$ are the 2 closest narrations in time to $y$. We work with batch containing $B$ positive video-narration pairs $\left\{\left(x_{i}, y_{i}\right)\right\}_{i \in[1, B]}$. We construct the set $\mathcal{N}$ by simply creating negative pairs from this batch by combining $\left\{\left(x_{i}, y_{j}\right)\right\}_{i \neq j}$. Since all representations are already computed, computing negative scores is cheap and efficient.

Optimization. We use the ADAM [41] optimizer with an initial learning rate of $10^{-3}$ with linear warm up of $5 \mathrm{k}$ steps. The learning rate is decayed twice by a factor of 10 . We train our model using Cloud TPUs v3 ${ }^{1}$, each Cloud TPU having a batch size of 128 videos. Given the high computational load required for training on HowTo100M, we

\footnotetext{
${ }^{1}$ https://cloud.google.com/tpu/
} 
(a) Training loss

\begin{tabular}{l|ccccc} 
Loss & YR10 & MR10 & CTR & HMDB & UCF \\
\hline Binary-Classif & 18.5 & 23.1 & 32.6 & 44.2 & 68.5 \\
Max margin & 16.3 & 24.1 & 29.3 & $\mathbf{5 6 . 2}$ & 76.6 \\
NCE & $\mathbf{2 9 . 1}$ & $\mathbf{2 7 . 0}$ & $\mathbf{3 5 . 6}$ & 55.4 & $\mathbf{7 7 . 5}$
\end{tabular}

(d) MIL strategy

\begin{tabular}{l|ccccc} 
Method & YR10 & MR10 & CTR & HMDB & UCF \\
\hline Cat+NCE & 31.9 & 30.8 & $\mathbf{3 5 . 2}$ & 56.3 & 78.9 \\
Max+NCE & 32.3 & 31.3 & 32.2 & 55.3 & 79.2 \\
Attn+NCE & 32.4 & 30.2 & 33.4 & 55.2 & 78.4 \\
MIL-NCE & $\mathbf{3 5 . 0}$ & $\mathbf{3 1 . 8}$ & 34.2 & $\mathbf{5 6 . 7}$ & $\mathbf{8 0 . 4}$
\end{tabular}

(b) Negatives per positive

\begin{tabular}{l|ccccc}
$\|\mathcal{N}\|$ & YR10 & MR10 & CTR & HMDB & UCF \\
\hline 64 & 26.0 & 25.5 & 33.1 & 56.1 & 76.0 \\
128 & 27.1 & 26.4 & 33.3 & $\mathbf{5 7 . 2}$ & 76.2 \\
256 & 28.7 & 28.7 & $\mathbf{3 6 . 5}$ & 56.5 & $\mathbf{7 7 . 5}$ \\
512 & $\mathbf{2 8 . 8}$ & $\mathbf{2 9 . 0}$ & 35.6 & 55.4 & 77.4
\end{tabular}

(e) Symmetric vs asymmetric negatives \begin{tabular}{l|l} 
Negatives & YR10 MR10 CTR HMDB UCF \\
\hline
\end{tabular} \begin{tabular}{l|lllll}
\hline$(x \mid y)$ & 34.4 & 29.0 & 33.9 & 55.1 & 78.1
\end{tabular} \begin{tabular}{l|lllll}
$(y \mid x)$ & 19.3 & 19.4 & 28.2 & $\mathbf{5 7 . 1}$ & 79.2
\end{tabular} \begin{tabular}{l|lllll}
$(x, y)$ & $\mathbf{3 5 . 0}$ & $\mathbf{3 1 . 8}$ & $\mathbf{3 4 . 2}$ & 56.7 & $\mathbf{8 0 . 4}$
\end{tabular} (c) Number of positive candidate pair

$$
\begin{array}{l|c|ccccc} 
& \text { NCE } & \multicolumn{5}{|c}{\text { MIL-NCE }} \\
\|\mathcal{P}\| \rightarrow & 1 & 3 & 5 & 9 & 17 & 33 \\
\hline \text { YR10 } & 29.1 & 33.6 & \mathbf{3 5 . 0} & 33.1 & 32.4 & 28.3 \\
\text { MR10 } & 27.0 & 30.2 & \mathbf{3 1 . 8} & 30.5 & 29.2 & 30.4 \\
\text { CTR } & 35.6 & \mathbf{3 7 . 3} & 34.2 & 31.8 & 25.0 & 25.0 \\
\text { HMDB } & 55.4 & \mathbf{5 7 . 8} & 56.7 & 55.7 & 54.8 & 51.4 \\
\text { UCF } & 77.5 & 79.7 & \mathbf{8 0 . 4} & 79.5 & 78.5 & 77.9
\end{array}
$$

(f) Language models

\begin{tabular}{l|ll} 
Text model & YR10 MR10 CTR HMDB UCF
\end{tabular}

\begin{tabular}{l|lllll}
\hline LSTM & 16.6 & 15.6 & 23.8 & 53.1 & 80.1
\end{tabular}

\begin{tabular}{l|lllll} 
GRU & 16.8 & 16.9 & 22.2 & 54.7 & $\mathbf{8 2 . 8}$
\end{tabular}

\begin{tabular}{l|lllll} 
Transformer & 26.7 & 26.5 & 32.7 & 53.4 & 78.4
\end{tabular}

\begin{tabular}{l|lllll} 
NetVLAD & 33.4 & 29.2 & $\mathbf{3 5 . 5}$ & 51.8 & 79.3
\end{tabular}

\begin{tabular}{l|lllll} 
Ours & $\mathbf{3 5 . 0}$ & $\mathbf{3 1 . 8}$ & 34.2 & $\mathbf{5 6 . 7}$ & 80.4
\end{tabular}

Table 2: Ablation studies

run ablation studies on 4 Cloud TPUs and train our model for 500k steps ( $\sim 3$ days). For our final evaluation in Section 4.4 , we pick the best parameters based on our ablation study and then use 64 Cloud TPUs for 400k steps (also $\sim 3$ days) as we observed that training on bigger batch size, and thus more epochs, had a positive impact on performance.

\subsection{Downstream tasks}

To show the generality of our learnt representations, we perform evaluation on five diverse downstream tasks using eight datasets described below.

Action Recognition: $H M D B-51$ [45], UCF-101 [69], Kinetics-700 [13]. We evaluate our video-only representation on the traditional HMDB-51 / UCF-101 as well as the recent Kinetics-700 action recognition tasks.

Text-to-Video retrieval: YouCook2 [90], MSR-VTT [87]. We use the YouCook2 and MSR-VTT text-to-video retrieval benchmarks to evaluate our off-the-shelf learnt joint text-video representation. We follow the same evaluation protocol as described in [52]. We report the retrieval performance using the recall at $\mathrm{K}(\mathrm{R} @ \mathrm{~K})$ metric (with $\mathrm{K}=1,5,10$ ) which measures the percentage of clips retrieved at the top $\mathrm{K}$ (the higher the better). We also report the median rank (MedR) of videos to be retrieved (the lower the better). Note from [52] that there is no intersection between YouCook2 testing and HowTo100M training videos.

Action Localization: YouTube-8M [1] Segments. We evaluate our video representation on YouTube-8M Segments ${ }^{2}$, a subset of the YouTube-8M [1] with precise temporal annotation. We follow the YouTube-8M Segments challenge evaluation protocol and report the mAP metric. ${ }^{3}$

Action Step Localization: CrossTask [91]. We use the recently released CrossTask instructional video dataset to

\footnotetext{
${ }^{2}$ https: //research.google.com/youtube $8 \mathrm{~m}$

3 https://www.kaggle.com/c/youtube8m-2019/ overview/evaluation
}

evaluate our off-the-shelf learnt joint text-video representation on the task of action step localization. We perform the same evaluation protocol as in [91] and report the average recall (CTR) metric for the localization task.

Action Segmentation: COIN [73]. We evaluate our videoonly representation on the COIN action segmentation task and follow the evaluation protocol of [71] by reporting the frame-wise accuracy (FA).

\subsection{Ablation studies}

We perform the ablation studies on the following downstream tasks: MSR-VTT R@10 (MR10), YouCook2 R@10 (YR10), HMDB-51 and UCF-101 recognition accuracy on split 1 and CrossTask average recall (CTR). This subset of downstream tasks has been chosen for their simplicity of evaluation and because they cover a wide range of tasks.

Which loss is better for learning the joint embedding?

In this ablation study (Table 2a), we compare different losses for matching the text and video embeddings in the standard single-instance learning setting where we pair each video clip to its closest narration in time. We compare the NCE based approach (ours) to the frequently used max margin ranking loss [18, 21, 33, 39, 51, 55, 79, 80, 83, 84] and a binary classification loss (i.e. sigmoid cross entropy loss) that has shown to be effective in video-audio matching $[6,7]$. Overall, the NCE loss outperforms other losses or works similarly on all five tested datasets.

The more negatives, the better. We keep the same singleinstance learning setting and assess the quality of our representations trained with different number of sampled negative examples per positive pair in Table $2 \mathrm{~b}$. We can see that the overall performance increases with the number of negatives. For the rest of the ablation studies, we use 512 negative samples per positive.

How many positive candidates pairs to consider? We evaluate the benefit of going from the single-instance learn- 

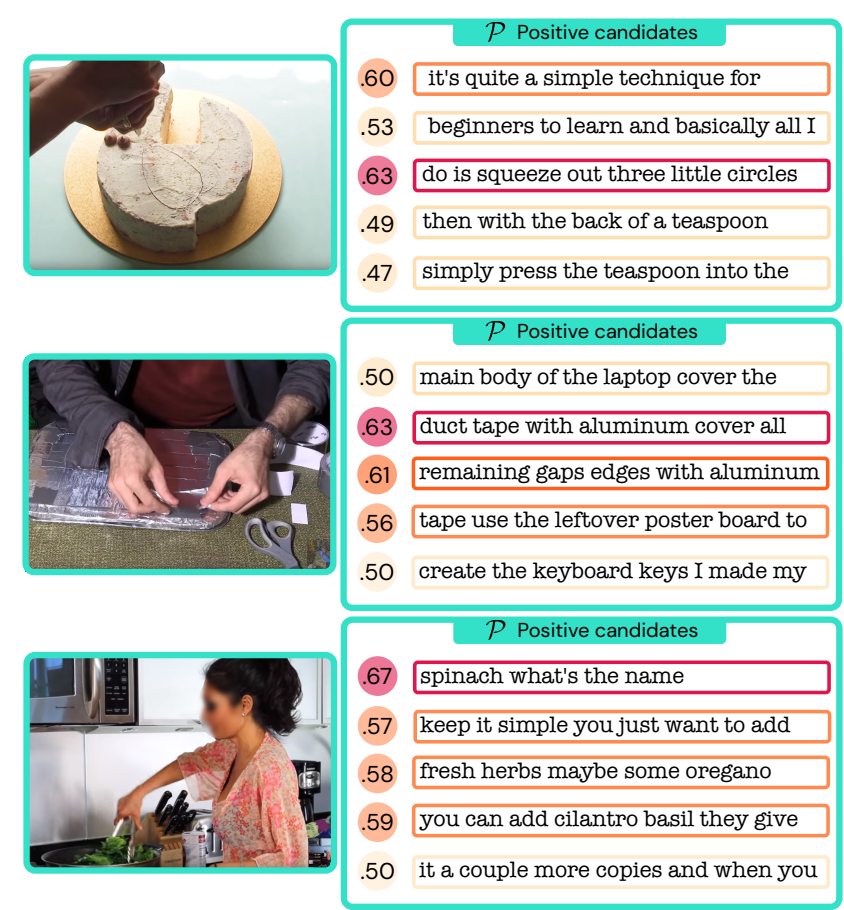

Figure 3: Selected video and narration pairs from five positive candidates on HowTo100M held-out samples using MIL-NCE.

ing approach to the proposed multiple-instance based approach in Table $2 \mathrm{c}$. In this experiment, we vary the number of positive candidate training pairs $\|\mathcal{P}\|$ for each video clip from 1 (i.e. single-instance learning setting) up to 33 candidates. Adding candidates significantly improves the performance upon the single-instance learning baseline. Moreover, we observe a trade-off between having too many candidates and not having enough of them, as we reach the best results by considering 3 to 5 positive candidates. We believe that adding too many contextual narrations increases the chance for irrelevant ones as they are sampled further in time from the considered video clip. For the rest of the paper we fix the number of positive candidate pairs to 5 .

MIL-NCE vs other MIL based approaches. In Table $2 \mathrm{~d}$, we compare our MIL-NCE approach with methods that can also handle multiple possible candidate captions at training time. The max-pool based approach $[4,7,58](\mathrm{Max}+\mathrm{NCE})$ only optimizes over the clip-caption pair with the highest similarity score among the positive candidates. On the other hand, the attention-based approach [36] (Attn+NCE) computes cross-modal attention weights between all the clipcaption pairs and perform a weighted average of the similarity scores in order to consider the most relevant positive candidate pairs. More details about these baselines are provided in the Appendix (section A). Finally, we also compare to the single-instance learning baseline where we concatenate all of the candidate narrations as one longer narration

\begin{tabular}{|c|c|c|c|c|c|c|}
\hline Method & Dataset & MM & Model & Frozen & HMDB & $\mathrm{UCF}$ \\
\hline OPN [46] & UCF & $x$ & VGG & $x$ & 23.8 & 59.6 \\
\hline Shuffle \& Learn [54]* & K600 & $x$ & S3D & $x$ & 35.8 & 68.7 \\
\hline Wang et al. [78] & K400 & Flow & C3D & $x$ & 33.4 & 61.2 \\
\hline CMC [74] & UCF & Flow & CaffeNet & $x$ & 26.7 & 59.1 \\
\hline Geometry [25] & $\mathrm{FC}$ & Flow & FlowNet & $x$ & 23.3 & 55.1 \\
\hline Fernando et al. [24] & UCF & $x$ & AlexNet & $x$ & 32.5 & 60.3 \\
\hline ClipOrder [86] & UCF & $x$ & $\mathrm{R}(2+1) \mathrm{D}$ & $x$ & 30.9 & 72.4 \\
\hline 3DRotNet [37]* & K600 & $x$ & S3D & $x$ & 40.0 & 75.3 \\
\hline DPC [30] & $\mathrm{K} 400$ & $x$ & 3D-R34 & $x$ & 35.7 & 75.7 \\
\hline 3D ST-puzzle [40] & K400 & $x$ & 3D-R18 & $x$ & 33.7 & 65.8 \\
\hline CBT [71] & K600 & $x$ & S3D & $\checkmark$ & 29.5 & 54.0 \\
\hline CBT [71] & K600 & $x$ & S3D & $x$ & 44.6 & 79.5 \\
\hline AVTS [43] & K600 & Audio & $\mathrm{I} 3 \mathrm{D}$ & $x$ & 53.0 & 83.7 \\
\hline AVTS [43] & Audioset & Audio & MC3 & $x$ & 61.6 & 89.0 \\
\hline \multirow{4}{*}{ Ours } & \multirow{4}{*}{ HTM } & \multirow{4}{*}{ Text } & \multirow{2}{*}{$\mathrm{I} 3 \mathrm{D}$} & $\checkmark$ & 54.8 & 83.4 \\
\hline & & & & $x$ & 59.2 & 89.1 \\
\hline & & & \multirow{2}{*}{ S3D } & $\checkmark$ & 53.1 & 82.7 \\
\hline & & & & $x$ & 61.0 & 91.3 \\
\hline \multicolumn{3}{|c|}{ Fully-supervised SOTA [85] } & S3D & $x$ & 75.9 & 96.8 \\
\hline
\end{tabular}

Table 3: Comparison to self-supervised methods on HMDB/UCF. Results are reported by averaging the accuracy over the 3 splits for both datasets. *Shuffle \& Learn and 3DRotNet reported numbers are reimplemented in [71] by using a better backbone (S3D). The $M M$ column indicates whether or not other modalities than the video frames have been used for the learning of the visual features. FC: FlyingChairs.

(Cat+NCE). Our proposed method outperforms these two standard approaches on five out of six tasks. Figure 3 illustrates examples of selected pairs from a hold-out set of HowTo100M videos, using MIL-NCE.

Symmetric or asymmetric negative sampling? Recall that given a pair of video/narration $(x, y)$, we create $\mathcal{N}$ in a symmetric manner by sampling negative narrations for the video $x$ and negative videos for the narration $y$. Table $2 \mathrm{e}$ compares that approach $(x, y)$ to asymmetric alternatives: (i) by fixing the video $x$ and only sampling negative captions $(y \mid x)$ and (ii) by fixing the narration $y$ and only sampling negative videos $(x \mid y)$. Overall, the best results are achieved when sampling jointly the negatives $(x, y)$, i.e. when we equally sample both video and narration negatives.

Which language model? Finally, we also experiment with different language models (1 layer LSTM [35] or GRU [17], 1 layer and 8 attention heads Transformer [75] and NetVLAD with 32 clusters [5]) and compare them to our simple model (see Table 1) in Table 2f. Even though our language model is similar to simple bag-of-word approach, it performs better on average and is more consistent over the five tasks than the other models. In particular, our model significantly outperforms the other language models on the text-to-video retrieval tasks (YR10 and MR10), where language plays the most important role. We believe that a sophisticated language understanding is not key for 


\begin{tabular}{lllc|l}
\multirow{2}{*}{ Method } & \multirow{2}{*}{ Net } & \multicolumn{2}{c|}{ Pretraining } & FA \\
& & Dataset & Labels & \\
\hline \multirow{3}{*}{ Ours } & R50 & ImNet & $\checkmark$ & 52.0 \\
& I3D & K400 & $\checkmark$ & 52.9 \\
CBT [71] & S3D & K600+HTM & $\checkmark$ & 53.9 \\
\hline Ours & I3D & HTM & $\boldsymbol{X}$ & $\mathbf{5 9 . 4}$ \\
Ours & S3D & HTM & $\boldsymbol{X}$ & $\mathbf{6 1 . 0}$
\end{tabular}

(a) COIN

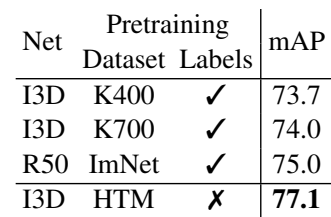

(b) YT8M-S

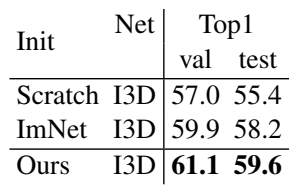

(c) $\mathrm{K} 700$

\begin{tabular}{lr|l} 
Method & Labels used & CTR \\
\hline Alayrac et al. [2] & ImNet+K400 & 13.3 \\
CrossTask [91] & ImNet+K400 & 22.4 \\
CrossTask [91] & ImNet+K400+CT & 31.6 \\
Miech et al. [52] & ImNet+K400 & 33.6 \\
\hline Ours (I3D) & None & $\mathbf{3 6 . 4}$ \\
Ours (S3D) & None & $\mathbf{4 0 . 5}$
\end{tabular}

(d) Cross Task (CT)

Table 4: Evaluation on action segmentation (a), localization (b, d) and recognition (c) benchmarks. K400: Kinetics-400, K600: Kinetics600, K700: Kinetics-700, HTM: HowTo100M, ImNet: ImageNet, YT8M-S: YouTube-8M Segments, R50: 2 D ResNet-50.

\begin{tabular}{llcccc} 
Method & Labeled dataset used & R@1 $\uparrow$ & R@5 & R@ $10 \uparrow$ & MedR $\downarrow$ \\
\hline Random & None & 0.03 & 0.15 & 0.3 & 1675 \\
HGLMM FV CCA [42] & ImNet + K400 + YouCook2 & 4.6 & 14.3 & 21.6 & 75 \\
Miech et al. [52] & ImNet + K400 & 6.1 & 17.3 & 24.8 & 46 \\
Miech et al. [52] & ImNet + K400 + YouCook2 & 8.2 & 24.5 & 35.3 & 24 \\
\hline Ours (I3D) & None & $\mathbf{1 1 . 4}$ & $\mathbf{3 0 . 6}$ & $\mathbf{4 2 . 0}$ & $\mathbf{1 6}$ \\
Ours (S3D) & None & $\mathbf{1 5 . 1}$ & $\mathbf{3 8 . 0}$ & $\mathbf{5 1 . 2}$ & $\mathbf{1 0}$
\end{tabular}

(a) YouCook2

\begin{tabular}{llcccc} 
Method & Labeled dataset used & R @ 1 $\uparrow$ & R $@ 5 \uparrow$ & R @ 10个 & MedR $\downarrow$ \\
\hline Random & None & 0.01 & 0.05 & 0.1 & 500 \\
Miech et al. [52] & ImNet + K400 & 7.5 & 21.2 & 29.6 & 38 \\
\hline Ours (I3D) & None & $\mathbf{9 . 4}$ & $\mathbf{2 2 . 2}$ & $\mathbf{3 0 . 0}$ & $\mathbf{3 5}$ \\
Ours (S3D) & None & $\mathbf{9 . 9}$ & $\mathbf{2 4 . 0}$ & $\mathbf{3 2 . 4}$ & $\mathbf{2 9 . 5}$
\end{tabular}

(b) MSRVTT

Table 5: Zero-shot evaluation on text-to-video retrieval.

our learning task. Instead, most of the time, detecting and matching the main keywords in each narration is enough. In Appendix (section C), we replace word2vec with a pretrained BERT model and obtain worse performance. We believe this is due to the domain gap between web text corpus and ASR outputs.

\subsection{Comparison to the state-of-the-art}

Video only representation. In Table 3 , we evaluate our learnt representations on the HMDB-51 [45] and UCF101 [69] action recognition benchmarks by extracting averaged pooled Mixed_5c features from the HowTo100M pretrained backbone. More specifically, we compare to selfsupervised approaches, which similarly to our work, do not make use of any annotated video or image dataset when training the visual representation. We outperform state-ofthe-art on UCF-101 and perform on par with AVTS [43] on HMDB-51. Most importantly, our learnt representation significantly outperforms many prior approaches even without fine-tuning. This result is significant as it demonstrates generalization of our representation to diverse sets of actions despite being trained on uncurated instructional videos.

Next, we evaluate our visual representation on COIN [73] action segmentation task in Table 4a. We split videos in subsequent clips of 1.5 seconds and represent them by concatenating three features: the local representation from I3D (or S3D), the global average pooled representation across the entire video and the relative positional embedding of the video clip. We train a logistic regression to predict the label for each clip. We compare our HowTo100M pretrained I3D network to an I3D fully-supervised on Kinetics-400, Kinetics-700 as well as a ResNet-50 fully supervised on ImageNet. We also compare to the state-of-the-art approach on COIN, CBT [71], which relies on a fully supervised S3D [85] trained on Kinetics600. Our learnt representation performs better than representations trained on Kinetics-400, Kinetics-700 or ImageNet. Moreover, our method also significantly outperforms the state-of-the-art CBT [71] despite their use of fully-supervised representation trained on Kinetics-600 and a Transformer model. We believe this improved localization ability is due to the fact that our model was trained on narrative descriptions with temporally localized timestamps as opposed to coarse video-level annotations.

We also report performance on the recently released YouTube-8M Segments dataset in Table 4b. Since no results have been published for this benchmark yet, we only compare the classification performance using different fullysupervised representations (i.e. I3D trained on Kinetics-400 / Kinetics-700 or ResNet-50 trained on ImageNet). Here again, our learnt representation outperforms all of the fullysupervised counterparts despite the domain gap between YouTube-8M and uncurated instructional videos.

Finally, in Table $4 \mathrm{c}$ we investigate the benefit of initializing an I3D model with our learned weights for a large-scale action recognition dataset (i.e. Kinetics-700).

We compare to a randomly initialized I3D and one inflated from an Inception network pretrained on ImageNet [14]. We obtain a $4 \%$ improvement over the randomly initialized I3D and $1.4 \%$ over the ImageNet pretrained I3D [14].

Joint text-video representation. We report text-to-video retrieval results on the YouCook2 (Table 5a) and MSRVTT (Table 5b) using our off-the-shelf model trained on HowTo100M. Note that our model has not seen any YouCook2 nor MSR-VTT annotated videos, hence for fair comparison on the MSR-VTT dataset we only compare to 
prior work [52] that did not finetune on MSR-VTT. On YouCook2, our model significantly outperforms all prior work. More specifically, it performs better than [52] which uses visual representation trained on Kinetics-400 + ImageNet and trains the joint text-video representation on both HowTo100M and YouCook2. On MSR-VTT, our method performs also better than [52], yet without using any manually annotated dataset. Finally, we also evaluate our off-theshelf model trained on HowTo100M on the CrossTask [91] action localization benchmark in Table 4d. We perform localization via a video-to-text retrieval approach similarly to [52]. Our method outperforms state-of-the-art approaches, here again, without using manual supervision.

\section{Conclusion}

We have addressed the challenging task of learning visual representations from scratch using uncurated instructional videos. Our approach did not rely on any manually annotated video or image dataset. To deal with the misalignment between narrations and videos, we have introduced MIL-NCE, a multiple instance learning approach derived from noise contrastive estimation. We have applied MIL-NCE to the uncurated HowTo100M dataset and obtained strong visual representations that outperformed selfsupervised as well as fully-supervised representations on downstream tasks. More generally, we believe MIL-NCE can be applicable in several multiple instance learning problems where representation learning is key.

Acknowledgements. We would like to thank: Relja Arandjelović, Pauline Luc, Gunnar Sigurdsson for helpful discussions. The project was partially supported by Antoine Miech Google PhD fellowship, the ERC grant LEAP (No.336845), the CIFAR Learning in Machines\&Brains program, and the European Regional Development Fund under the project IMPACT (reg. no. CZ.02.1.01/0.0/0.0/15_003/0000468).

\section{References}

[1] Sami Abu-El-Haija, Nisarg Kothari, Joonseok Lee, Paul Natsev, George Toderici, Balakrishnan Varadarajan, and Sudheendra Vijayanarasimhan. YouTube-8M: A largescale video classification benchmark. arXiv preprint arXiv:1609.08675, 2016. 2, 6

[2] Jean-Baptiste Alayrac, Piotr Bojanowski, Nishant Agrawal, Ivan Laptev, Josef Sivic, and Simon Lacoste-Julien. Unsupervised learning from narrated instruction videos. In $C V P R$, 2016. 3,8

[3] Elad Amrani, Rami Ben-Ari, Tal Hakim, and Alex Bronstein. Toward self-supervised object detection in unlabeled videos. arXiv preprint arXiv:1905.11137, 2019. 3
[4] Stuart Andrews, Ioannis Tsochantaridis, and Thomas Hofmann. Support vector machines for multiple-instance learning. In NIPS, 2003. 3, 7

[5] Relja Arandjelović, Petr Gronat, Akihiko Torii, Tomas Pajdla, and Josef Sivic. NetVLAD: CNN architecture for weakly supervised place recognition. In $C V P R, 2016.7$

[6] Relja Arandjelović and Andrew Zisserman. Look, listen and learn. In $I C C V, 2017.6$

[7] Relja Arandjelović and Andrew Zisserman. Objects that sound. In ECCV, 2018. 2, 6, 7

[8] Boris Babenko, Ming-Hsuan Yang, and Serge Belongie. Visual tracking with online multiple instance learning. In CVPR, 2009. 3

[9] Francis Bach and Zaïd Harchaoui. DIFFRAC: a discriminative and flexible framework for clustering. In NIPS, 2007. 3

[10] Angie Boggust, Kartik Audhkhasi, Dhiraj Joshi, David Harwath, Samuel Thomas, Rogerio Feris, Dan Gutfreund, Yang Zhang, Antonio Torralba, Michael Picheny, et al. Grounding spoken words in unlabeled video. In $C V P R W, 2019.3$

[11] Piotr Bojanowski, Francis Bach, Ivan Laptev, Jean Ponce, Cordelia Schmid, and Josef Sivic. Finding Actors and Actions in Movies. In ICCV, 2013. 3

[12] Mathilde Caron, Piotr Bojanowski, Julien Mairal, and Armand Joulin. Unsupervised pre-training of image features on non-curated data. In ICCV, 2019. 2

[13] João Carreira, Eric Noland, Chloe Hillier, and Andrew Zisserman. A short note on the kinetics-700 human action dataset. arXiv preprint arXiv:1907.06987, 2019. 6

[14] João Carreira and Andrew Zisserman. Quo vadis, action recognition? a new model and the kinetics dataset. In $C V P R$, 2017. 2, 3, 5, 8

[15] Kai Chen, Hang Song, Chen Change Loy, and Dahua Lin. Discover and learn new objects from documentaries. In CVPR, 2017. 3

[16] Guilhem Chéron, Jean-Baptiste Alayrac, Ivan Laptev, and Cordelia Schmid. A flexible model for training action localization with varying levels of supervision. In NeurIPS, 2018. 3

[17] Kyunghyun Cho, Bart van Merrienboer, Dzmitry Bahdanau, and Yoshua Bengio. On the Properties of Neural Machine Translation: Encoder-Decoder Approaches. arXiv preprint arXiv:1409.1259, 2014. 7

[18] Mithun Chowdhury, Panda Rameswar, Evangelos Papalexakis, and Amit Roy-Chowdhury. Webly supervised joint embedding for cross-modal image-text retrieval. In ACM International Conference on Multimedia, 2018. 3, 6

[19] Jacob Devlin, Ming-Wei Chang, Kenton Lee, and Kristina Toutanova. BERT: Pre-training of deep bidirectional transformers for language understanding. In $A C L, 2019.14$

[20] Thomas G Dietterich, Richard H Lathrop, and Tomás Lozano-Pérez. Solving the multiple instance problem with axis-parallel rectangles. Artificial intelligence, 89(1-2):3171, 1997. 3

[21] Jianfeng Dong, Xirong Li, Chaoxi Xu, Shouling Ji, Yuan He, Gang Yang, and Xun Wang. Dual encoding for zero-example video retrieval. In CVPR, 2019. 3, 6 
[22] Olivier Duchenne, Ivan Laptev, Josef Sivic, Francis Bach, and Jean Ponce. Automatic annotation of human actions in video. In $I C C V, 2009.3$

[23] Debidatta Dwibedi, Yusuf Aytar, Jonathan Tompson, Pierre Sermanet, and Andrew Zisserman. Temporal cycleconsistency learning. In $C V P R, 2019.2$

[24] Basura Fernando, Hakan Bilen, Efstratios Gavves, and Stephen Gould. Self-supervised video representation learning with odd-one-out networks. In CVPR, 2017. 2, 7

[25] Chuang Gan, Boqing Gong, Kun Liu, Hao Su, and Leonidas J Guibas. Geometry guided convolutional neural networks for self-supervised video representation learning. In $C V P R, 2019.2,7$

[26] Deepti Ghadiyaram, Du Tran, and Dhruv Mahajan. Largescale weakly-supervised pre-training for video action recognition. In CVPR, 2019. 2

[27] Yunchao Gong, Qifa Ke, Michael Isard, and Svetlana Lazebnik. A multi-view embedding space for modeling internet images, tags, and their semantics. IJCV, 2014. 3

[28] Yunchao Gong, Liwei Wang, Micah Hodosh, Julia Hockenmaier, and Svetlana Lazebnik. Improving image-sentence embeddings using large weakly annotated photo collections. In $E C C V$, 2014. 3

[29] Michael Gutmann and Aapo Hyvärinen. Noise-contrastive estimation: A new estimation principle for unnormalized statistical models. In AISTATS, 2010. 3, 5

[30] Tengda Han, Weidi Xie, and Andrew Zisserman. Video representation learning by dense predictive coding. arXiv preprint arXiv:1909.04656, 2019. 2, 7

[31] David Harwath, Adrià Recasens, Dídac Surís, Galen Chuang, Antonio Torralba, and James Glass. Jointly discovering visual objects and spoken words from raw sensory input. In $E C C V, 2018.3$

[32] Olivier J Hénaff, Ali Razavi, Carl Doersch, SM Eslami, and Aaron van den Oord. Data-efficient image recognition with contrastive predictive coding. arXiv preprint arXiv:1905.09272, 2019. 5

[33] Lisa Anne Hendricks, Oliver Wang, Eli Shechtman, Josef Sivic, Trevor Darrell, and Bryan Russell. Localizing moments in video with natural language. ICCV, 2017. 6

[34] Jack Hessel, Bo Pang, Zhenhai Zhu, and Radu Soricut. A case study on combining asr and visual features for generating instructional video captions. arXiv preprint arXiv:1910.02930, 2019. 3

[35] Sepp Hochreiter and Jurgen Schmidhuber. Long short-term memory. In Neural Computing, 1997. 7

[36] Maximilian Ilse, Jakub M Tomczak, and Max Welling. Attention-based deep multiple instance learning. arXiv preprint arXiv:1802.04712, 2018. 7

[37] Longlong Jing and Yingli Tian. Self-supervised spatiotemporal feature learning by video geometric transformations. arXiv preprint arXiv:1811.11387, 2018. 2, 7

[38] Rafal Jozefowicz, Oriol Vinyals, Mike Schuster, Noam Shazeer, and Yonghui Wu. Exploring the limits of language modeling. arXiv preprint arXiv:1602.02410, 2016. 5

[39] Andrej Karpathy, Armand Joulin, and Fei Fei F Li. Deep fragment embeddings for bidirectional image sentence mapping. In NIPS, 2014. 6
[40] Dahun Kim, Donghyeon Cho, and In So Kweon. Selfsupervised video representation learning with space-time cubic puzzles. In $A A A I, 2019.7$

[41] Diederik P. Kingma and Jimmy Ba. Adam: A method for stochastic optimization. In ICLR, 2015. 5

[42] Benjamin Klein, Guy Lev, Gil Sadeh, and Lior Wolf. Associating neural word embeddings with deep image representations using fisher vectors. In CVPR, 2015. 3, 8

[43] Bruno Korbar, Du Tran, and Lorenzo Torresani. Cooperative learning of audio and video models from self-supervised synchronization. In NeurIPS, 2018. 2, 7, 8

[44] Hilde Kuehne, Ahsan Iqbal, Alexander Richard, and Juergen Gall. Mining youtube-a dataset for learning fine-grained action concepts from webly supervised video data. arXiv preprint arXiv:1906.01012, 2019. 3

[45] Hildegard Kuehne, Hueihan Jhuang, Estíbaliz Garrote, Tomaso Poggio, and Thomas Serre. HMDB: a large video database for human motion recognition. In ICCV, 2011. 6, 8

[46] Hsin-Ying Lee, Jia-Bin Huang, Maneesh Singh, and MingHsuan Yang. Unsupervised representation learning by sorting sequences. In ICCV, 2017. 2, 7

[47] Thomas Leung, Yang Song, and John Zhang. Handling label noise in video classification via multiple instance learning. In $I C C V, 2011.3$

[48] Tsung-Yi Lin, Michael Maire, Serge Belongie, James Hays, Pietro Perona, Deva Ramanan, Piotr Dollár, and C Lawrence Zitnick. Microsoft COCO: Common Objects in Context. In ECCV, 2014. 3

[49] Jonathan Malmaud, Jonathan Huang, Vivek Rathod, Nick Johnston, Andrew Rabinovich, and Kevin Murphy. What's cookin'? interpreting cooking videos using text, speech and vision. NAACL, 2015. 3

[50] Antoine Miech, Jean-Baptiste Alayrac, Piotr Bojanowski, Ivan Laptev, and Josef Sivic. Learning from Video and Text via Large-Scale Discriminative Clustering. In ICCV, 2017. 3

[51] Antoine Miech, Ivan Laptev, and Josef Sivic. Learning a Text-Video Embedding from Incomplete and Heterogeneous Data. arXiv preprint arXiv:1804.02516, 2018. 3, 6

[52] Antoine Miech, Dimitri Zhukov, Jean-Baptiste Alayrac, Makarand Tapaswi, Ivan Laptev, and Josef Sivic. Howto100M: Learning a text-video embedding by watching hundred million narrated video clips. In ICCV, 2019. 1, 2, $3,4,5,6,8,9$

[53] Tomas Mikolov, Kai Chen, Greg Corrado, and Jeffrey Dean. Efficient estimation of word representations in vector space. arXiv preprint arXiv:1301.3781, 2013. 5

[54] Ishan Misra, C Lawrence Zitnick, and Martial Hebert. Shuffle and learn: unsupervised learning using temporal order verification. In $E C C V$, 2016. 2, 7

[55] Niluthpol Chowdhury Mithun, Juncheng Li, Florian Metze, and Amit K Roy-Chowdhury. Learning joint embedding with multimodal cues for cross-modal video-text retrieval. In ICMR. ACM, 2018. 3, 6

[56] Yasufumi Moriya, Ramon Sanabria, Florian Metze, and Gareth JF Jones. Grounding object detections with transcriptions. arXiv preprint arXiv:1906.06147, 2019. 3 
[57] Aaron van den Oord, Yazhe Li, and Oriol Vinyals. Representation learning with contrastive predictive coding. arXiv preprint arXiv:1807.03748, 2018. 5

[58] Maxime Oquab, Leon Bottou, Ivan Laptev, and Josef Sivic. Is object localization for free? - weakly-supervised learning with convolutional neural networks. In CVPR, June 2015. 7

[59] Shruti Palaskar, Jindrich Libovickỳ, Spandana Gella, and Florian Metze. Multimodal abstractive summarization for how2 videos. arXiv preprint arXiv:1906.07901, 2019. 3

[60] Yingwei Pan, Tao Mei, Ting Yao, Houqiang Li, and Yong Rui. Jointly modeling embedding and translation to bridge video and language. In $C V P R, 2016.3$

[61] Omkar Parkhi, Esa Rahtu, and Andrew Zisserman. It's in the bag: Stronger supervision for automated face labelling. In ICCV Workshop, 2015. 3

[62] Bryan A Plummer, Matthew Brown, and Svetlana Lazebnik. Enhancing video summarization via vision-language embedding. In $C V P R, 2017.3$

[63] Bryan A Plummer, Liwei Wang, Chris M Cervantes, Juan C Caicedo, Julia Hockenmaier, and Svetlana Lazebnik. Flickr30k entities: Collecting region-to-phrase correspondences for richer image-to-sentence models. In ICCV, 2015. 3

[64] Vignesh Ramanathan, Armand Joulin, Percy Liang, and Li Fei-Fei. Linking people in videos with "their" names using coreference resolution. In ECCV, 2014. 3

[65] Anna Rohrbach, Atousa Torabi, Marcus Rohrbach, Niket Tandon, Christopher Pal, Hugo Larochelle, Aaron Courville, and Bernt Schiele. Movie description. IJCV, 2017. 3

[66] Olga Russakovsky, Jia Deng, Hao Su, Jonathan Krause, Sanjeev Satheesh, Sean Ma, Zhiheng Huang, Andrej Karpathy, Aditya Khosla, Michael Bernstein, Alexander C. Berg, and Li Fei-Fei. ImageNet Large Scale Visual Recognition Challenge. IJCV, 2015. 3

[67] Ramon Sanabria, Ozan Caglayan, Shruti Palaskar, Desmond Elliott, Loïc Barrault, Lucia Specia, and Florian Metze. How2: a large-scale dataset for multimodal language understanding. In Proceedings of the Workshop on Visually Grounded Interaction and Language (ViGIL). NeurIPS, 2018. 2, 3

[68] Nataliya Shapovalova, Arash Vahdat, Kevin Cannons, Tian Lan, and Greg Mori. Similarity constrained latent support vector machine: An application to weakly supervised action classification. In ECCV, 2012. 3

[69] Khurram Soomro, Amir Roshan Zamir, and Mubarak Shah. UCF101: A dataset of 101 human actions classes from videos in the wild. arXiv preprint arXiv:1212.0402, 2012. 6,8

[70] Waqas Sultani, Chen Chen, and Mubarak Shah. Real-world anomaly detection in surveillance videos. In CVPR, 2018. 3

[71] Chen Sun, Fabien Baradel, Kevin Murphy, and Cordelia Schmid. Contrastive bidirectional transformer for temporal representation learning. arXiv preprint arXiv:1906.05743, 2019. 2, 3, 6, 7, 8

[72] Chen Sun, Austin Myers, Carl Vondrick, Kevin Murphy, and Cordelia Schmid. Videobert: A joint model for video and language representation learning. In ICCV, 2019. 3
[73] Yansong Tang, Dajun Ding, Yongming Rao, Yu Zheng, Danyang Zhang, Lili Zhao, Jiwen Lu, and Jie Zhou. Coin: A large-scale dataset for comprehensive instructional video analysis. In $C V P R, 2019.6,8$

[74] Yonglong Tian, Dilip Krishnan, and Phillip Isola. Contrastive multiview coding. arXiv preprint arXiv:1906.05849, 2019. 2,7

[75] Ashish Vaswani, Noam Shazeer, Niki Parmar, Jakob Uszkoreit, Llion Jones, Aidan N Gomez, Łukasz Kaiser, and Illia Polosukhin. Attention is all you need. In NIPS, 2017. 7

[76] Carl Vondrick, Hamed Pirsiavash, and Antonio Torralba. Anticipating visual representations from unlabeled video. In CVPR, 2016. 2

[77] Carl Vondrick, Abhinav Shrivastava, Alireza Fathi, Sergio Guadarrama, and Kevin Murphy. Tracking emerges by colorizing videos. In $E C C V, 2018.2$

[78] Jiangliu Wang, Jianbo Jiao, Linchao Bao, Shengfeng He, Yunhui Liu, and Wei Liu. Self-supervised spatio-temporal representation learning for videos by predicting motion and appearance statistics. In CVPR, 2019. 2, 7

[79] Liwei Wang, Yin Li, Jing Huang, and Svetlana Lazebnik. Learning two-branch neural networks for image-text matching tasks. PAMI, 2018. 3, 6

[80] Liwei Wang, Yin Li, and Svetlana Lazebnik. Learning deep structure-preserving image-text embeddings. In $C V P R$, 2016. 3, 6

[81] Xiaolong Wang and Abhinav Gupta. Unsupervised learning of visual representations using videos. In ICCV, 2015. 2

[82] Philippe Weinzaepfel, Xavier Martin, and Cordelia Schmid. Towards weakly-supervised action localization. arXiv preprint arXiv:1605.05197, 2016. 3

[83] Michael Wray, Diane Larlus, Gabriela Csurka, and Dima Damen. Fine-grained action retrieval through multiple partsof-speech embeddings. In ICCV, 2019. 3, 6

[84] Chao-Yuan Wu, R Manmatha, Alexander J Smola, and Philipp Krähenbühl. Sampling matters in deep embedding learning. ICCV, 2017. 3, 6

[85] Saining Xie, Chen Sun, Jonathan Huang, Zhuowen Tu, and Kevin Murphy. Rethinking spatiotemporal feature learning: Speed-accuracy trade-offs in video classification. In $E C C V$, 2018. 5, 7, 8

[86] Dejing Xu, Jun Xiao, Zhou Zhao, Jian Shao, Di Xie, and Yueting Zhuang. Self-supervised spatiotemporal learning via video clip order prediction. In CVPR, 2019. 2, 7

[87] Jun Xu, Tao Mei, Ting Yao, and Yong Rui. Msr-vtt: A large video description dataset for bridging video and language. In CVPR, 2016. 3, 6

[88] Ran Xu, Caiming Xiong, Wei Chen, and Jason J Corso. Jointly modeling deep video and compositional text to bridge vision and language in a unified framework. In $A A A I, 2015$. 3

[89] Shoou-I Yu, Lu Jiang, and Alexander Hauptmann. Instructional videos for unsupervised harvesting and learning of action examples. In ACM, 2014. 3

[90] Luowei Zhou, Chenliang Xu, and Jason J Corso. Towards automatic learning of procedures from web instructional videos. In $A A A I$, 2018. 3, 6 
[91] Dimitri Zhukov, Jean-Baptiste Alayrac, Ramazan Gokberk Cinbis, David Fouhey, Ivan Laptev, and Josef Sivic. Crosstask weakly supervised learning from instructional videos. In $C V P R, 2019.6,8,9$ 


\section{Appendix overview}

We provide in Section A technical details about the baselines introduced in Table 2 (d - MIL strategy) from the ablation studies. Finally Section B provides a visualization of the model architecture used in our work.

\section{A. Max+NCE and Attn+NCE baselines}

We use the same notation as in the main paper for this section.

Max+NCE. This baseline aims at reproducing the standard max-pool based approach often used in multiple instance learning, but here combined with the NCE loss. Formally, this can be written as maximizing the following objective:

$$
\max _{f, g} \sum_{i=1}^{n} \log \left(\mathrm{MaxNCE}_{i}\right)
$$

where:

$$
\operatorname{MaxNCE}_{i}=\frac{\max _{(x, y) \in \mathcal{P}_{i}} e^{f(x)^{\top} g(y)}}{\max _{(x, y) \in \mathcal{P}_{i}} e^{f(x)^{\top} g(y)}+\sum_{\left(x^{\prime}, y^{\prime}\right) \sim \mathcal{N}_{i}} e^{f\left(x^{\prime}\right)^{\top} g\left(y^{\prime}\right)}} .
$$

Intuitively, this corresponds to choosing the best positive candidate pair among all pairs $\mathcal{P}_{i}$ according to the model.

Attn+NCE. This other baseline aims at selecting best candidate pairs via a cross-modal soft-attention mechanism between the clips and narrations. The cross-modal attention mechanism $a$ is defined as follows:

$$
a\left(x, y, \mathcal{P}_{i}\right)=\frac{e^{f_{a}(x)^{\top} g_{a}(y)}}{\sum_{\left(x^{\prime}, y^{\prime}\right) \in \mathcal{P}_{i}} e^{f_{a}\left(x^{\prime}\right)^{\top} g_{a}\left(y^{\prime}\right)}},
$$

where $f_{a}$ and $g_{a}$ are two parametrized functions. In practice $f_{a}$ and $g_{a}$ are sharing parameters with $f$ (respectively $g$ ) except for the last 'Linear' layer (see Figure 4). Given that cross-modal attention mechansim, the Attn+NCE objective is:

$$
\max _{f, g, a} \sum_{i=1}^{n} \log \left(\operatorname{AttnNCE}_{i}\right)
$$

where:

$$
\operatorname{AttnNCE}_{i}=\frac{\sum^{(x, y) \in \mathcal{P}_{i}}{ }_{e^{(x, y) \in \mathcal{P}_{i}}} a\left(x, y, \mathcal{P}_{i}\right) f(x)^{\top} g(y)}{\sum_{\left(x^{\prime}, y^{\prime}\right) \sim \mathcal{N}_{i}} a\left(x, y \mathcal{P}_{i}\right) f(x)^{\top} g(y)}+.
$$

The intuition behind this approach is to allow the model to have a separate selection mechanism for the positive candidate pairs.

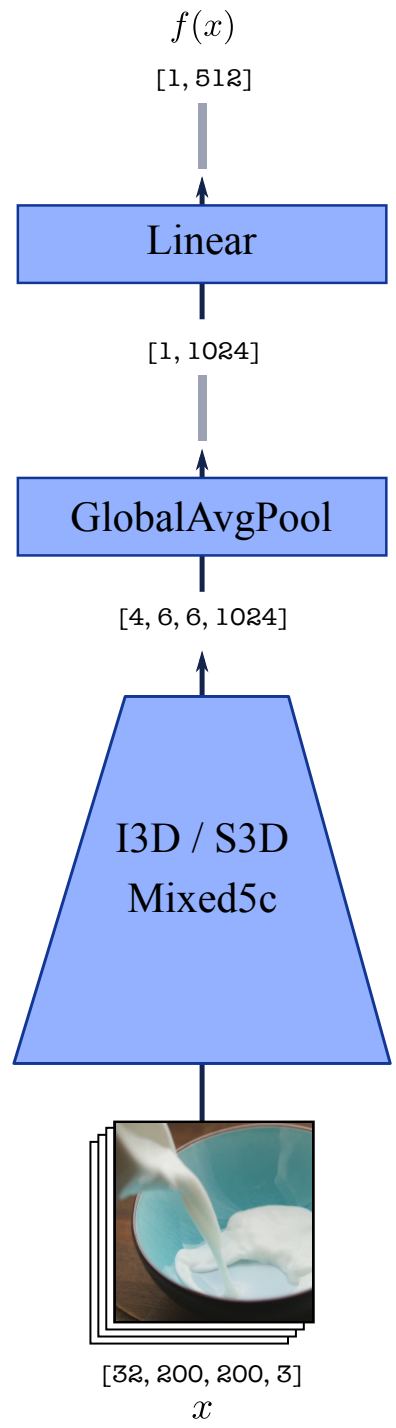

\section{Video model}

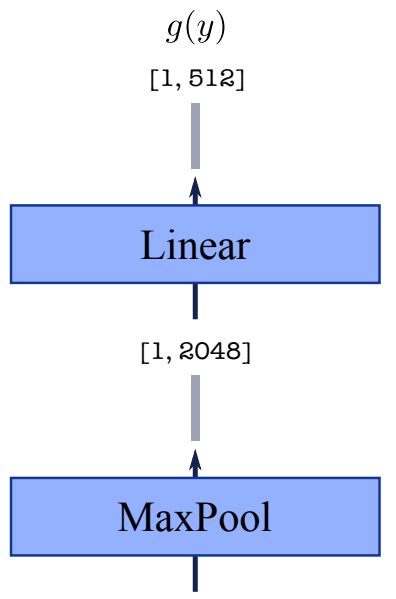

$[16,2048]$

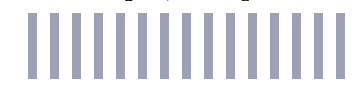

$\uparrow$

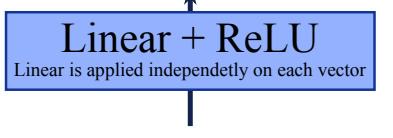

$[16,300]$

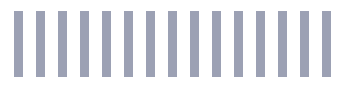

1

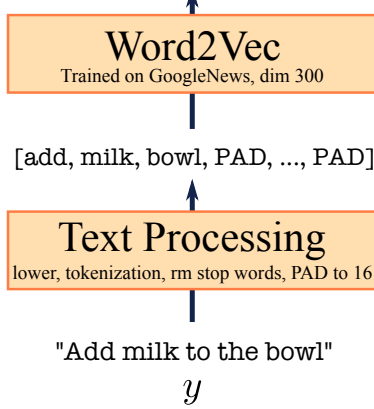

Text model

Figure 4: Model architecture. In this figure, we provide a visualization of the video embedding network $f$ (left) and the text embedding network $g$ (right). Modules displayed in blue are trained from scratch on the challenging uncurated HowTo100M dataset using the MIL-NCE loss. The word embeddings are learned in an unsupervised fashion using Word2Vec trained on GoogleNews and are kept fixed during training. Finally, the dimensions of the outputs of each layer are specified in brackets, e.g. the output of the 'Word2Vec' layer is of size $[16,300]$ corresponding to the 16 word embedding vectors of dimension 300 (one vector for each word, also materialized by the 16 grey rectangles). 


\begin{tabular}{lcc}
\hline Input word vectors & YR10 & MR10 \\
\hline BERT wo. stop words & 19.0 & 19.7 \\
BERT w. stop words & 17.6 & 23.9 \\
Word2Vec & $\mathbf{3 5 . 0}$ & $\mathbf{3 1 . 8}$ \\
\hline
\end{tabular}

Table 6: Results when using BERT vectors as inputs instead of Word2vec.

\section{B. Model architecture}

Figure 4 provides an illustration of the video model $f$ and text model $g$ used in the main paper.

\section{Pretrained BERT}

We provide an ablation study where we replace the input text vectors coming from Word2Vec with more advanced contextual word embedding vectors from a BERT model [19]. In details, we use the BERT base model ${ }^{4}$ to replace the Word2Vec module in our model (see Figure B): we process the sequence of 16 input words to obtain 16 output vectors of dimension 768. Apart from the fact that the input dimension of word vectors is increased from 300 to 768 , the rest of the text model is kept the same. For a fair comparison in terms of number of parameters, we do not finetune the BERT model. For this experiment, we use an I3D model under the same training setting used in the ablation study 4.3. Also, as BERT may be sensitive to the absence of stop words, we also run an experiment where we do not remove the stop words during the text preprocessing phase. Results are given in Table 6. We observe a strong degradation in performance when using BERT pretrained vectors. We believe this is due to the domain gap between web text corpus and ASR outputs. We leave further investigation as future work.

\footnotetext{
${ }^{4}$ https://tfhub.dev/google/bert_cased_L-12_ H-768_A-12/1
} 